\author{
Marquette University \\ e-Publications@Marquette
}

\title{
Measurement and Modeling of Infrared Nonlinear Absorption Coefficients and Laser-induced Damage Thresholds in Ge and $\mathrm{GaSb}$
}

\section{T. J. Wagner}

Air Force Institute of Technology

M. A. Bohn

Air Force Institute of Technology

Ronald A. Coutu Jr.

Marquette University, ronald.coutu@marquette.edu

L. P. Gonzalez

Air Force Research Laboratory, Materials and Manufacturing Directorate

J. M. Murray

Air Force Research Laboratory, Materials and Manufacturing Directorate

See next page for additional authors

Follow this and additional works at: https://epublications.marquette.edu/electric_fac

Part of the Computer Engineering Commons, and the Electrical and Computer Engineering Commons

\section{Recommended Citation}

Wagner, T. J.; Bohn, M. A.; Coutu, Ronald A. Jr.; Gonzalez, L. P.; Murray, J. M.; Schepler, K. L.; and Guha, S., "Measurement and Modeling of Infrared Nonlinear Absorption Coefficients and Laser-induced Damage Thresholds in Ge and GaSb" (2010). Electrical and Computer Engineering Faculty Research and Publications. 346.

https://epublications.marquette.edu/electric_fac/346 


\section{Authors}

T. J. Wagner, M. A. Bohn, Ronald A. Coutu Jr., L. P. Gonzalez, J. M. Murray, K. L. Schepler, and S. Guha 


\title{
Measurement and modeling of infrared nonlinear absorption coefficients and laser-induced damage thresholds in Ge and GaSb
}

\author{
T. J. Wagner, ${ }^{1, *}$ M. J. Bohn, ${ }^{1}$ R. A. Coutu, Jr., ${ }^{1}$ L. P. Gonzalez, ${ }^{2}$ J. M. Murray, ${ }^{2}$ K. L. Schepler, ${ }^{3}$ and S. Guha ${ }^{2}$ \\ ${ }^{1}$ Air Force Institute of Technology, Wright Patterson Air Force Base, Ohio 45433, USA \\ ${ }^{2}$ Air Force Research Laboratory, Materials and Manufacturing Directorate, Wright Patterson Air Force Base, \\ Ohio 45433, USA \\ ${ }^{3}$ Air Force Research Laboratory, Sensors Directorate, Wright Patterson Air Force Base, Ohio 45433, USA \\ *Corresponding author: torrey.wagner@us.af.mil
}

Received July 20, 2010; accepted July 28, 2010;

posted August 6, 2010 (Doc. ID 131970); published September 23, 2010

\begin{abstract}
Using a simultaneous fitting technique to extract nonlinear absorption coefficients from data at two pulse widths, we measure two-photon and free-carrier absorption coefficients for Ge and GaSb at 2.05 and $2.5 \mu \mathrm{m}$ for the first time, to our knowledge. Results agreed well with published theory. Single-shot damage thresholds were also measured at $2.5 \mu \mathrm{m}$ and agreed well with modeled thresholds using experimentally determined parameters including nonlinear absorption coefficients and temperature dependent linear absorption. The damage threshold for a single-layer $\mathrm{Al}_{2} \mathrm{O}_{3}$ anti-reflective coating on Ge was $55 \%$ or $35 \%$ lower than the uncoated threshold for picosecond or nanosecond pulses, respectively.

OCIS codes: $190.0190,140.3330,350.1820$.
\end{abstract}

\section{INTRODUCTION}

Since the invention of the laser 50 years ago [1], incrementally shorter pulses and higher $2-3 \mu \mathrm{m}$ middleinfrared (mid-IR) pulse energies have been demonstrated [2], resulting in high peak irradiances that can cause significant changes in optical materials. As is well known and also demonstrated here, the nonlinear absorption (NLA) of a material can change from less than $5 \%$ to greater than $90 \%$ based solely on the level of incident irradiance. The absorbed energy can lead to a rise of temperature above the melting point, which is why the subjects of NLA and damage are intertwined. As higher peak power mid-IR laser sources are developed, it will be crucial to measure the NLA of transmissive mid-IR materials.

There were three specific objectives in this research involving mid-IR laser effects. The first was to measure the NLA coefficients of Ge and GaSb at 2.05 and $2.5 \mu \mathrm{m}$. These materials can be used in mid-IR sensors and were also selected to study the difference in NLA and laserinduced damage between direct bandgap ( $\mathrm{GaSb})$ and indirect bandgap (Ge) semiconductors. The second objective was to model the dynamics of NLA that lead to surface temperature rise and eventually thermal damage from a single laser pulse, including temperature dependent linear absorption $\alpha(T)$ and carrier density dependent recombination $\tau_{R}(N)$. The final objective was to test the laserinduced damage threshold (LIDT) due to these effects and to compare with modeling.

\section{THEORY}

Two-photon absorption (TPA) was first proposed in 1931 by Nobel laureate Maria Göppert-Mayer [3]. However,
TPA could not be confirmed in the optical frequency range until the demonstration of the laser. In 1961, TPA was first observed in $\mathrm{CaF}_{2}: \mathrm{Eu}^{2+}$ crystals at a wavelength of $694 \mathrm{~nm}$ by Kaiser and Garrett [4].

Degenerate TPA was studied in this work, where two photons of equal energy are absorbed to create one electron-hole pair, but it is also possible to study nondegenerate TPA using two beams of differing frequencies. The atomic-level effects that contribute to TPA and its polarization response are described in $[5,6]$. The TPA coefficient $\beta$ can be predicted from the bandgap $E_{g}$, photon energy $E_{p}$, Kane parameter $K$, and refractive index $n$ using Van Stryland's empirical simplification to Wherrett's scaling law, which is presented in Eq. (1) $[7,8]$. For an indirect bandgap material such as Ge, predictions of $\beta$ use the bandgap at the $\Gamma$ point, as $\beta$ is 2000 times smaller at the indirect gap, and therefore the interaction at the indirect gap can be ignored [9]:

$$
\begin{gathered}
\beta(\omega)=K \frac{\sqrt{E_{p}}}{n^{2}(\omega) E_{g}{ }^{3}} F_{2}\left(\frac{h \nu}{E_{g}}\right), \\
x=\frac{h \nu}{E_{g}} .
\end{gathered}
$$

Free-carrier absorption (FCA) is an optical transition where a photon is absorbed by an excited carrier (electron or hole) and is measured by the FCA cross section $\sigma . \sigma$ depends on wavelength and is related to the free-carrier density $N$ by the expression $\alpha_{\mathrm{FCA}}=\sigma N$, where $\alpha_{\mathrm{FCA}}$ denotes linear absorption due to free carriers. This absorption can occur from intrinsic or excited carriers that include free electrons in the conduction band and free holes 
Table 1. Survey of Nonlinear Studies for Germanium, Ordered by Wavelength ${ }^{a}$

\begin{tabular}{|c|c|c|c|c|c|}
\hline $\begin{array}{c}\lambda \\
(\mu \mathrm{m})\end{array}$ & Pulse Width & $\begin{array}{l}\operatorname{TPA}(\beta) \\
(\mathrm{cm} / \mathrm{GW})\end{array}$ & $\begin{array}{l}\mathrm{FCA}(\sigma) \\
\left(\mathrm{cm}^{2}\right)\end{array}$ & $\begin{array}{l}\text { Dopant Level } \\
\quad\left(\mathrm{cm}^{-3}\right)\end{array}$ & Reference \\
\hline 2.36 & $\dagger$ & 1000 & - & $\dagger$ & [12] \\
\hline $2.6-3.1$ & $100 \mathrm{~ns}$ & 2500 & - & $\dagger$ & [13] \\
\hline 2.65 & $480 \mathrm{~ns}$ & 680 & - & $n \sim 10^{15}-10^{16}$ & [14] \\
\hline 2.80 & $480 \mathrm{~ns}$ & 325 & - & $n \sim 10^{15}-10^{16}$ & [14] \\
\hline 2.90 & $2 \mathrm{ps}$ & $80 \pm 10$ & $6 \pm 1 \times 10^{-17 *}$ & $\dagger$ & [15] \\
\hline 2.95 & $480 \mathrm{~ns}$ & 100 & - & $n \sim 10^{15}-10^{16}$ & [14] \\
\hline 3.00 & $2 \mathrm{ps}$ & $20 \pm 5$ & $6 \pm 1 \times 10^{-17 *}$ & $\dagger$ & [15] \\
\hline
\end{tabular}

$a_{\dagger}=$ not given, $-=$ not measured, ${ }^{*}=$ non-degenerate value measured using a $2.9 \mu \mathrm{m}$ pump and a $3 \mu \mathrm{m}$ probe.

in the valence band. This is a cumulative nonlinearity as there is a lifetime associated with the carriers. The free carriers will continue to absorb until they recombine, which occurs at a rate that is both material dependent and free-carrier density dependent. A full band-structure study indicates that the variation of FCA with wavelength is not strictly monotonically increasing [10].

The optically and thermally excited carriers generated by linear absorption and NLA induce many processes that affect the level of absorption, heat rise $(T)$, and generated free-carrier density $(N)$ in a material and causes radiation to propagate according to Eqs. (2)-(5) [11]. Experimentally measured temperature dependent linear absorption $\alpha(T)$ is presented later in Fig. 2 and results in greater accuracy by coupling Eq. (4) into Eqs. (2) and (3). Additionally, the inclusion of free-carrier density dependent recombination $\tau_{R}(N)$ in Eq. (5) was required as $N$ varied over 6 orders of magnitude during damage threshold modeling:

$$
\begin{gathered}
\frac{d I}{d z}=-\alpha(T) I-\beta I^{2}-\sigma\left(N_{0}+N\right) I, \\
\frac{d N}{d t}=\frac{\alpha(T) I}{h \nu}+\frac{\beta I^{2}}{2 h \nu}-\frac{N}{\tau_{r}(N)}, \\
\frac{d T}{d t}=\frac{\alpha(T) I}{\rho C}+\frac{\beta I^{2}}{\rho C}+\frac{\sigma\left(N_{0}+N\right) I}{\rho C}, \\
\frac{1}{\tau_{R}}=B_{\text {radiative }} N(r, z, t)+C_{\text {auger }} N(r, z, t)^{2} .
\end{gathered}
$$

In Eqs. (2)-(5), $I, \sigma, N_{0}, \tau_{R}, \rho$, and $C$ are irradiance, FCA coefficient, intrinsic free-carrier density, free-carrier recombination time, material density, and specific heat, respectively. $r, z$, and $t$ denote variation in sample radial position, sample length, and time.

Previously published results of NLA near 2.05 and $2.5 \mu \mathrm{m}$ wavelengths in Ge are shown in Table 1 . The nearest literature wavelengths for Ge are 2.36 and $2.6 \mu \mathrm{m}$, but those studies did not account for FCA. The next closest study occurred at $2.8 \mu \mathrm{m}$, where Eq. (1) predicts that TPA is reduced by $60 \%$ when compared with a wavelength of $2.5 \mu \mathrm{m}$.

$\mathrm{GaSb}$ is far less studied, and there is only one paper in which TPA was measured. In a 1996 work, Akmanov et al. used an estimated $\sigma=2 \times 10^{-17} \mathrm{~cm}^{2}$ to measure $\beta=380 \mathrm{~cm} / \mathrm{GW}$ in GaSb at a wavelength of $2.94 \mu \mathrm{m}$ [16]. At a wavelength of $2.05 \mu \mathrm{m}$, a $\beta$ of $156 \mathrm{~cm} / \mathrm{GW}$ was reported as a theoretical calculation for GaSb [17].

\section{A. Laser-Induced Damage Study}

The LIDT of a material is influenced by many simultaneous wavelength-dependent processes that operate on widely varying time scales. For picosecond and longer pulses, the LIDT can vary with linear absorption, NLA, and several material properties including heat capacity, thermal conductivity, and carrier recombination rates. The processes that affect damage at femtosecond time scales are very different, transitioning from thermal mechanisms to dielectric breakdown as the extreme peak irradiance resulting from the ultrashort pulse duration can cause the energy to be deposited faster than free carriers can relax into phonons [18]. The literature was reviewed for prior work, and while there were no damage studies performed on GaSb, two Ge studies are reported in Table 2.

The $0.25 \mu \mathrm{m}$ study occurs in the linear absorption regime, and the $2.8 \mu \mathrm{m}$ test is the only study where TPA is the dominant damage mechanism [20]. The longer wavelengths in the 2008 study rely on three-photon absorption (3PA) and four-photon absorption and lead to dielectric breakdown. Additionally, the 2.8-5.2 $\mu \mathrm{m}$ tests were conducted on a multiple pulse free-electron laser, not a single shot test as performed in this work.

An implicit finite difference (FD) numerical method was used to solve Eqs. (2)-(5) and was used both to extract NLA coefficients and to model temperature rise resulting in laser-induced damage. The pulse and sample were broken up into elements in time, sample radius, and sample length, using the following assumptions:

- The incident beam has radial symmetry and contains quasi-monochromatic light.

- The sample is thin compared to the beam confocal parameter, resulting in negligible diffraction while traversing the sample, and nonlinear refraction is insignificant.

- The slowly varying envelope and paraxial approximations can be applied.

- Three-photon (and higher) absorption and tunneling ionization are insignificant.

- The temperature dependences of $\beta, \sigma$, and $\tau_{R}$ are insignificant. 
Table 2. Survey of Damage Studies for Germanium, Ordered By Wavelength

\begin{tabular}{|c|c|c|c|c|c|}
\hline $\begin{array}{c}\lambda \\
(\mu \mathrm{m})\end{array}$ & Pulse Width & $\begin{array}{l}\text { LIDT } \\
\left(\mathrm{J} / \mathrm{cm}^{2}\right)\end{array}$ & $\begin{array}{l}\text { Dopant Level } \\
\quad\left(\mathrm{cm}^{-3}\right)\end{array}$ & $\begin{array}{l}\text { Beam Spot Radius } \\
\qquad(\mu \mathrm{m})\end{array}$ & Reference \\
\hline 0.25 & $38 \mathrm{~ns}$ & 0.33 & $p=10^{17}$ & 1500 & [19] \\
\hline 2.8 & $5 \mu \mathrm{sEL}^{a}$ & 5.3 & $n=10^{13}-10^{14}$ & $260-380$ & [20] \\
\hline 3.2 & $5 \mu \mathrm{SEL}^{a}$ & 12.8 & $n=10^{13}-10^{14}$ & $260-380$ & {$[20]$} \\
\hline 3.6 & $5 \mu \mathrm{sEL}^{a}$ & 21.5 & $n=10^{13}-10^{14}$ & $260-380$ & [20] \\
\hline 4.0 & $5 \mu \mathrm{SEL}^{a}$ & 22.5 & $n=10^{13}-10^{14}$ & $260-380$ & [20] \\
\hline 4.4 & $5 \mu \mathrm{sEL}^{a}$ & 26.0 & $n=10^{13}-10^{14}$ & $260-380$ & {$[20]$} \\
\hline 4.8 & $5 \mu \mathrm{SEL}^{a}$ & 24.8 & $n=10^{13}-10^{14}$ & $260-380$ & [20] \\
\hline 5.2 & $5 \mu \mathrm{SEL}^{a}$ & 22.8 & $n=10^{13}-10^{14}$ & $260-380$ & [20] \\
\hline
\end{tabular}

${ }^{a}$ Free-electron laser- $-30 \mathrm{~Hz}, 5 \mu$ s length macropulse consisting of 10,000 1 ps micropulses.

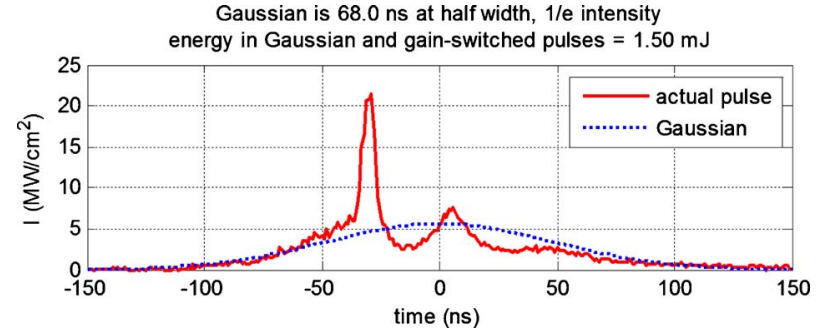

Fig. 1. (Color online) Gain-switched temporal profile (solid trace) with Gaussian best fit overlay (dotted trace).

- The spot radius $r_{o}$ and pulse repetition frequency (PRF) are chosen to avoid free-carrier diffusion and thermal diffusion.

An advantage of breaking the pulse into elements is that a non-Gaussian temporal profile can be implemented, which allows the accurate modeling of nonlinear coefficients even if the input pulse has a unusual time profile, which is typical of gain-switched lasers [21,22]. When appropriate, the FD model either used a Gaussian profile or was modified to accept the measured temporal profile from a gain-switched $\mathrm{Cr}^{2+}: \mathrm{ZnSe}$ nanosecond laser. The repeatable temporal profile from this source is shown in Fig. 1 (jagged trace), and an equivalent Gaussian pulse was fit to the gain-switched pulse, resulting in an admittedly poor best fit of $68 \mathrm{~ns} \tau_{p}$.

It is clear that the peak irradiance of the gain-switched pulse is much greater than an equivalent Gaussian. Modeling indicated that if a $5 \mathrm{~mJ}$ pulse were incident on a $265 \mu \mathrm{m}$ spot radius, the resulting temperature rise from this gain-switched profile would melt the surface of a Ge sample while an equivalent Gaussian pulse would leave the surface undamaged. The energy in a non-Gaussian pulse can be expressed as $E=A r e a_{\text {spot }} \int I d t$ or alternatively $E=$ Area $_{\text {spot }} \Sigma\left(I_{i} * \tau\right)$, where $\tau$ is the oscilloscope time step of $0.2 \mathrm{~ns}$. The irradiance profile $I_{i}$ in Fig. 1 was determined by multiplying the oscilloscope voltage trace $V_{i}$ from a $1.5 \mathrm{~mJ}$ pulse by a constant $M$, and varying $M$ until $1.5 \mathrm{~mJ}=$ Area $_{\text {spot }} \Sigma\left(V_{i} M^{*} \tau\right)$. Using this method and a $265 \mu \mathrm{m}$ spot radius at $1 / e$ irradiance, the peak irradiance was calculated as $22.3 \mathrm{MW} / \mathrm{cm}^{2}$.

\section{SEMICONDUCTOR CHARACTERIZATION}

In order to measure NLA coefficients and model damage thresholds, accurate knowledge of the optical, electrical, and thermal properties of a material is required. For Ge and $\mathrm{GaSb}$, these properties are presented in Table 3 from either measurement or the literature. Two second-order effects that influence NLA and damage are modeled, which are variation of linear absorption with temperature and variation of free-carrier recombination time with free-carrier density. The effectiveness of an anti-reflective coating is also reported.

Temperature dependent absorption: In order to increase the accuracy of damage modeling, temperature dependent Fourier transform infrared spectra for Ge and GaSb were obtained [23]. The goal of this effort was to capture the increase in linear absorption $(\alpha)$ that occurs due to lattice expansion and increased phonon density from thermally excited carriers. As shown by the black traces in Fig. 2, linear absorption at $2.5 \mu \mathrm{m}$ increases dramatically at temperatures above $350 \mathrm{~K}(450 \mathrm{~K})$ for $\mathrm{Ge}$ (GaSb). The red traces represent $\alpha(T)$ for both materials that resulted from the experiment.

The material bandgap $E_{g}$ also changes with temperature [23], which will modify the NLA by altering $\beta$ as shown in Eq. (1). However, $E_{g}(T)$ and the resulting $\beta(T)$ are not implemented in the FD model as the resulting $\Delta \beta$ was calculated to be less than the error bars on the final measurements. If the band edge approaches the photon energy due to the bandgap shift, the $\alpha(T)$ measurement would capture any increase in linear absorption from this effect.

Free-carrier density dependent recombination: FCA will decrease if a significant number of free carriers recombine during a pulse. Since free carriers relax at a rate that depends on the free-carrier density $N$, this effect was studied as it can significantly affect FCA, transmission, and temperature rise leading to damage. Auger recombination, radiative recombination, and Shockley-ReadHall (SRH) recombination all contribute; however SRH was ignored as its microsecond lifetimes far exceed the picosecond/nanosecond $\tau_{p}$ in this work [24,25]. $N$-dependent recombination lifetimes $\tau_{R}(N)$ are included in the FD model and were calculated for the peak $N$ in the NLA tests. When the recombination rates in Table 3 are used to calculate $\tau_{R}$ from Eq. (5), an important difference between indirect and direct bandgap materials is highlighted. For an equivalent $N$, radiative recombination is suppressed in Ge as it must be phonon assisted, and resulted in $\tau_{R}(N)$ of 250-500 ns during the NLA testing. This was several times longer than the $37-68 \mathrm{~ns}$ pulses in 
Table 3. Material Properties for Ge and GaSb Samples

\begin{tabular}{|c|c|c|c|c|}
\hline Property & $\mathrm{Ge}$ & Ref. $^{a}$ & $\mathrm{GaSb}$ & Ref. $^{a}$ \\
\hline Thickness (mm) & 3.05 & M & 1.06 & M \\
\hline Index of refraction at $2.5 \mu \mathrm{m}$ & 4.06 & {$[26]$} & 3.749 & {$[26]$} \\
\hline Fresnel loss per surface $(\%)$ & 37 & $\mathrm{C}$ & 34 & $\mathrm{C}$ \\
\hline Bandgap at the $\Gamma$ point $(\mathrm{eV})$ & 0.800 & {$[26]$} & 0.726 & {$[26]$} \\
\hline Surface roughness $R_{a}$ parameter $(\mathrm{nm})$ & 1.73 & M & 1.46 & M \\
\hline Surface roughness $R_{q}$ parameter $(\mathrm{nm})$ & 2.21 & $\mathrm{M}$ & 1.87 & M \\
\hline Melting temperature $\left({ }^{\circ} \mathrm{K}\right)$ & 1210 & {$[26]$} & 985 & {$[26]$} \\
\hline Thermal conductivity $(\mathrm{W} / \mathrm{cm} \mathrm{K})$ & 0.58 & {$[26]$} & 0.32 & {$[26]$} \\
\hline Density $\left(\mathrm{g} / \mathrm{cm}^{3}\right)$ & 5.32 & {$[26]$} & 5.61 & {$[26]$} \\
\hline Specific heat $\left(\mathrm{J} \mathrm{g}^{-1} \mathrm{~K}^{-1}\right)$ & 0.31 & {$[26]$} & 0.25 & {$[26]$} \\
\hline Thermal diffusivity $\left(\mathrm{cm}^{2} / \mathrm{s}\right)$ & 0.352 & {$[26]$} & 0.228 & {$[26]$} \\
\hline Free-carrier diffusivity $\left(\mathrm{cm}^{2} / \mathrm{s}\right)$ & 44 & {$[27]$} & 77 & {$[28]$} \\
\hline Resistivity $(\Omega \mathrm{cm})$ & 1865 & M & 2717 & M \\
\hline Intrinsic free-carrier density $\left(\mathrm{cm}^{-3}\right)$ & $\sim 10^{13}$ & $\mathrm{C}$ & $\sim 10^{12}$ & $\mathrm{C}$ \\
\hline Electron mobility $\mu_{n}\left(\mathrm{~cm}^{2} / \mathrm{V} \mathrm{s}\right)$ & 3900 & {$[26]$} & 3000 & [26] \\
\hline Hole mobility $\mu_{p}\left(\mathrm{~cm}^{2} / \mathrm{V} \mathrm{s}\right)$ & 1900 & {$[26]$} & 1000 & {$[26]$} \\
\hline Ionizing potential $(\mathrm{eV})$ & 7.9 & {$[29]$} & 5.03 & {$[30]$} \\
\hline Radiative recombination rate $\left(\mathrm{cm}^{3} / \mathrm{s}\right)$ & $6.4 \times 10^{-14}$ & {$[26]$} & $1.2 \times 10^{-9}$ & $\mathrm{M}$ \\
\hline Auger recombination rate $\left(\mathrm{cm}^{6} / \mathrm{s}\right)$ & $1 \times 10^{-30}$ & {$[26]$} & $5 \times 10^{-30}$ & [17] \\
\hline
\end{tabular}

${ }^{a}$ Legend: $\mathrm{M}=$ measured, $\mathrm{C}=$ calculated.

these experiments, and $\tau_{R}$ did not reduce NLA in Ge. However, the GaSb $\tau_{R}(N)$ of 45-65 ns was on the order of the pulse width, which reduced the NLA and increased the LIDT as carrier recombination occurred during the pulse.

The high refractive indices of $\mathrm{Ge}$ and $\mathrm{GaSb}$ result in significant Fresnel reflections at each surface, which would cause strong internal reflections that could distort the NLA measurements. The transmission was increased to $80 \%-90 \%$ by applying a single layer $360 \mathrm{~nm}$ aluminum oxide $\left(\mathrm{Al}_{2} \mathrm{O}_{3}\right)$ anti-reflective coating to a portion of each sample.

Surface inspections before testing were performed on an atomic force microscope (Veeco, NanoScope V). The results from this measurement are presented in Table 3 and show that GaSb has slightly lower surface roughness. Table 3 also lists Ge and GaSb optical, electrical, and thermal properties used in this work.

\section{PROCESSES IMPACTING NLA MEASUREMENTS}

There are several factors that contribute to nonlinear absorption and damage, and accurate measurement of nonlinear coefficients requires careful test design to avoid effects that cannot easily be modeled.

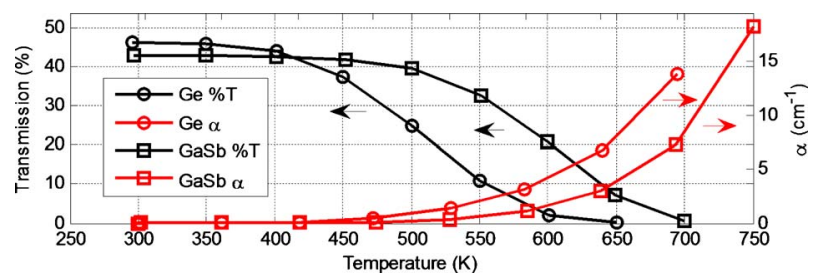

Fig. 2. (Color online) $2.5 \mu \mathrm{m}$ variation of linear transmission (left axis) and $\alpha$ (right axis) with temperature.
Thermal diffusion: Heat generated by the incident pulse can alter the level of linear absorption as shown in Fig. 2. Test results could be distorted if heat escapes during the pulse width or if residual heat builds up in the spot area over repeated pulses. To avoid these effects, test constraints for spot size and maximum laser PRF are calculated. The distance that heat will diffuse within the time of the laser pulse is the definition of the thermal diffusion length $L$ [31]. For a $70 \mathrm{~ns}$ pulse width, the thermal diffusion length for Ge and GaSb is less than $4 \mu \mathrm{m}$ and will not affect the experiments in this work, but may need to be taken into account for microsecond and longer pulses.

If testing is accomplished using a train of pulses, thermal diffusion dictates a maximum PRF that will allow heat to dissipate out of the spot area in between pulses. This PRF is calculated using the expression $\tau_{d}=r_{o}{ }^{2} / 4 D$ with a spot radius $r_{o}$, diffusivity $D$, and diffusion time $\tau_{d}$ [32]. The maximum $\mathrm{PRF}$ is calculated as a repetition rate whose period is ten times $\tau_{d}$ so that any heat generated can escape the area in between pulses. A $700 \mu \mathrm{m}$ spot radius would require a maximum PRF of 29 or $19 \mathrm{~Hz}$ for $\mathrm{Ge}$ and $\mathrm{GaSb}$, respectively.

Free-carrier diffusion: In addition to recombining, free carriers generated by the incident pulse can diffuse out of the spot area, which would reduce FCA and therefore alter the NLA. Using the same method as thermal diffusion, a free-carrier diffusion length can be calculated using a Ge value of $D=44 \mathrm{~cm}^{2} / \mathrm{s}$, and $D=77 \mathrm{~cm}^{2} / \mathrm{s}$ for GaSb $[27,28]$. Free-carrier diffusion is much faster than thermal diffusion, but will still be insignificant for picosecond pulses. However, for a $70 \mathrm{~ns}$ pulse width, free-carrier diffusion lengths of 35 and $46 \mu \mathrm{m}$ were calculated for Ge and $\mathrm{GaSb}$, respectively. Care was taken to choose spot sizes larger than the free-carrier diffusion length in the nanosecond experiments.

Intrinsic free carrier absorption: Modeling indicated 
that $N_{0}$ could influence absorption if the sample is heavily doped, and $N_{0}$ was measured using a resistivity method to rule out this effect. The high resistivities of 1900 or $2700 \Omega \mathrm{cm}$ for $\mathrm{Ge}$ and $\mathrm{GaSb}$, respectively, indicate that the samples are of high purity with a calculated level of intrinsic carrier density between $10^{12}$ and $10^{13} \mathrm{~cm}^{-3} \cdot N_{0}$ was not used in the FD model as levels $<10^{15} \mathrm{~cm}^{-3}$ were insignificant compared to the carrier density generated by the pulse and had no impact on the results.

Nonlinear refraction: Refraction can be induced in a material from nonlinear refraction, thermal lensing, or free-carrier refraction, based on instantaneous, thermal, or cumulative effects, respectively. There are two ways that nonlinear refraction could affect the nonlinear measurements. The first is that induced refraction could cause the beam to be defocused to the point that some of the pulse energy misses the detector, resulting in an inaccurate energy measurement. This potential problem was alleviated by placing the energy head close to the sample exit surface in order to collect all transmitted energy.

The second issue is that nonlinear refraction could focus or defocus the beam within the sample, varying the irradiance and therefore the level of NLA. Refraction would have the greatest effect on the Ge sample as its thickness was three times greater than the GaSb sample, and its value of $d n / d T$ is ten times greater. In order to rule out refractive effects, modeling was performed on the Ge NLA data collected in this work at a wavelength of $2.05 \mu \mathrm{m}$ using a numerical model that incorporated diffraction effects [33]. Nominal values of Ge nonlinear refraction $n_{2}=7 \times 10^{-13} \mathrm{~cm}^{2} / \mathrm{W}$, thermo-optic coefficient $d n / d T=400 \times 10^{-6} \mathrm{~K}^{-1}$, and free-carrier refraction cross section $\sigma_{F C R}=7 \times 10^{-21} \mathrm{~cm}^{3}$ resulted in less than $0.1 \%$ transmission change, due to thin samples and less than $4 \mathrm{~K}$ temperature rise during testing.

Three-photon absorption: Although the bandgaps of $\mathrm{Ge}$ and GaSb would permit 3PA at wavelengths of 2.05 and $2.5 \mu \mathrm{m}$, it was assumed to be negligible at the irradiances used in this study as there is a significantly lower probability associated with $3 \mathrm{PA}$ in a regime where TPA is possible [34].

\section{EXPERIMENT AND RESULTS}

This section describes six experiments and their results that measure and model nonlinear optical absorption leading to damage in semiconductors. First, NLA coefficients are measured using an irradiance scan method [33], and then damage thresholds that result from NLA are measured at two pulse widths. Finally, LIDT modeling was performed using measured NLA coefficients, $\tau_{R}(N)$ and $\alpha(T)$, in order to compare theory to experiment and explore the pulse width dependence of the LIDT.

The uncertainty in measured nonlinear coefficient values is proportional to $I^{2}$. In order to minimize experimental uncertainty, the incident beam was carefully measured, and the apparatus was aligned and calibrated before and after testing, referencing the irradiance scan apparatus in Fig. 3. Attenuation to achieve a range of irradiances across 2 orders of magnitude was accomplished

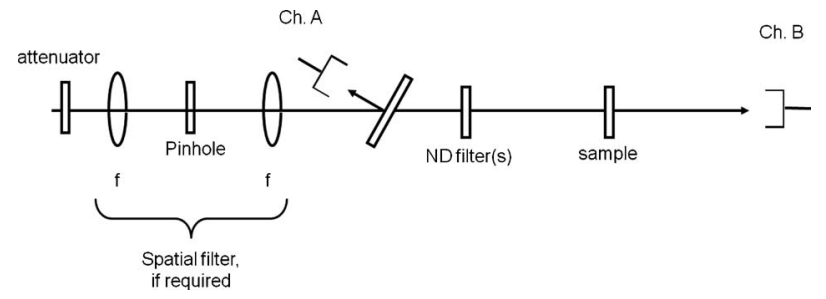

Fig. 3. NLA and damage testing experimental setup.

with a computer controlled attenuator stage (Newport, PR50PP) and two neutral density filters that were flipped in and out of the beam.

As discussed above, there is wide variation in reported $\beta$ values. While the majority of the variation was due to the exclusion of FCA effects, some variation could be attributed to imprecise definitions of peak pulse irradiance, pulse width, and spot size. In this work, Gaussian spatial profiles are verified, the spot size $r_{o}$ was defined as the radius at the 1/e level of irradiance, $\tau_{p}$ was defined as the pulse half-width at $1 / e$ irradiance, and the peak pulse irradiance was derived as $I_{0}=E /\left(\sqrt{\pi} \tau_{p} \pi r_{o}{ }^{2}\right)$, where $E$ is the pulse energy. This derivation of Gaussian peak pulse irradiance results when the definitions above are used and radial symmetry is assumed. In each experiment a $10 \mu \mathrm{m}$ pinhole was used to measure the beam radius and verify a Gaussian spatial profile along $x$ and $y$ dimensions. Temporal duration was measured using an autocorrelator for the picosecond laser source, and mid-IR fast photodetectors for the nanosecond laser sources.

In experiments I and II, NLA data were collected on Ge and GaSb at wavelengths of 2.05 and $2.5 \mu \mathrm{m}$ using picosecond pulses. The laser source consisted of $10 \mathrm{~Hz}$ modelocked Nd:ytrrium aluminum garnet (Ekspla, PL2143) and difference frequency generator (Ekspla, DFG2-10P) that produced the desired wavelengths. The beam was spatially filtered to achieve a Gaussian spatial profile. A non-collinear autocorrelator was used to measure the $2.5 \mu \mathrm{m}$ pulse width as $10.1 \pm 0.83 \mathrm{ps}$, where a Gaussian temporal profile was assumed. The same technique measured the $2.05 \mu \mathrm{m}$ pulse width as $10.2 \pm 0.8 \mathrm{ps}$. For the $2.5 \mu \mathrm{m}$ NLA tests, a $734 \mu \mathrm{m}$ spot radius at $1 / e$ irradiance was measured, and a $584 \mu \mathrm{m}$ spot radius was measured for the $2.05 \mu \mathrm{m}$ NLA tests. NLA data are presented in the simultaneous fit section that follows the experimental descriptions.

Experiment III was conducted on Ge and GaSb using a nanosecond $2.05 \mu \mathrm{m}$ Tm,Ho:YLF (yttrium lithium fluoride) laser. This source has been described elsewhere [21]; however, the laser was designed for continuous-wave (CW) or kilohertz $Q$-switched operation which was not suitable for NLA testing. In order to satisfy the thermal diffusion constraint on PRF, a quasi-CW pumping scheme was implemented to give $10 \mathrm{~Hz}$ operation with an optimal $Q$-switch delay based on the work of Louchev et al. $[35,36]$. The spatial and temporal profiles were very well approximated by Gaussians, with measured values of $\tau_{p}$ $=37 \mathrm{~ns}$ and $r_{o}=375 \mu \mathrm{m}$ in $x$ and $y$ axes.

Experiment IV was conducted on $\mathrm{Ge}$ and $\mathrm{GaSb}$ at a wavelength of $2.47 \mu \mathrm{m}$ using nanosecond pulses from a gain-switched $\mathrm{Cr}^{2+}: \mathrm{ZnSe}$ laser. The source was characterized to a $265 \mu \mathrm{m}$ spot radius at focus with a near- 

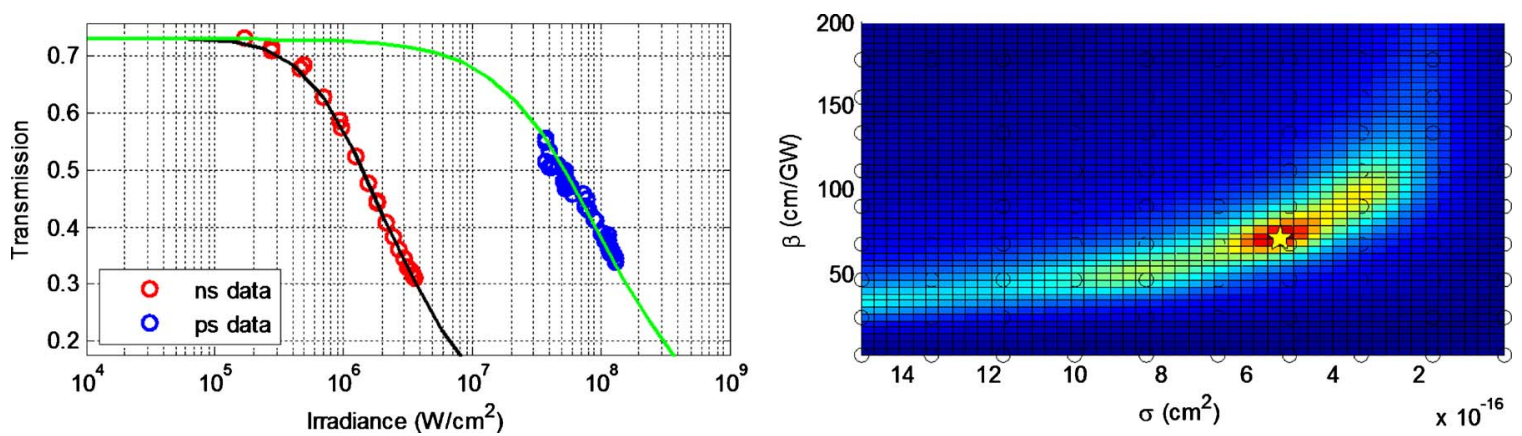

Fig. 4. (Color online) Ge $2.05 \mu \mathrm{m}$ fitting routine. The left graph presents nanosecond NLA data (circles in left trace) and picosecond data (circles in right trace) along with FD model output using the best fit pair of $\beta$ and $\sigma$. This pair is determined from the right graph in which the yellow star signifies the minimum error.

Gaussian spatial profile, and the temporal profile was presented in Fig. 1. The spectral content of the pulses was measured using a monochromator (ARC, SpectraPro750), yielding a $2.47 \mu \mathrm{m}$ peak emission and $110 \mathrm{~nm}$ linewidth. The picosecond experiment was performed using $2.50 \mu \mathrm{m}$ pulses, so there was a slight difference in wavelengths. Using Eq. (1), $\beta$ theoretically changes by less than $4 \%$ across the range $2.4-2.51 \mu \mathrm{m}$ for either Ge or GaSb, which was acceptable as it was less than the error bars on the final $\beta$ measurements.

Simultaneous fit to NLA coefficients: A quantitative simultaneous fit method was performed on all NLA data in order to extract $\beta$ and $\sigma$, which are difficult to separate using other methods. This builds on the work in [37], where $\beta$ and $\sigma$ are measured using a self-consistent method on dual-pulse width NLA data. In this subsection the motivation for this method is presented, the fitting method is described, $\beta$ and $\sigma$ are measured for Ge and GaSb at wavelengths of 2.05 and $2.5 \mu \mathrm{m}$, and an uncertainty analysis is performed.

As $\sigma$ is a cumulative nonlinearity, conventional wisdom has held that $\beta$ can be isolated with an ultrashort pulse. That pulse width was derived in a recent work [38], which states that the pulse width must satisfy $\tau_{p} \ll 2 h \nu \beta L / \sigma$ to isolate $\beta$, where $L$ is the sample length. The $10 \mathrm{ps}$ pulse width experiments in this work do not satisfy this condition for either the Ge or GaSb sample, which would require $\tau_{p} \ll 45 \mathrm{fs}$. The fact that the pulses were too long to isolate $\beta$ was evident in the simultaneous $\beta$ and $\sigma$ fits shown in this study.

A $\beta$ measurement can vary by orders of magnitude if FCA is not included, especially for nanosecond and longer pulses. This was shown in a 1973 study where NLA was observed in a $5.1 \mathrm{~mm}$ thick uncoated germanium [13]. The laser output covered a spectrum from 2.6-3.1 $\mu \mathrm{m}$, pulse width was $100 \mathrm{~ns}$, and peak irradiances ranged from $1-10 \mathrm{MW} / \mathrm{cm}^{2}$. FCA was not used, and $\beta$ alone was used to fit the transmission, resulting in $\beta=2500 \mathrm{~cm} / \mathrm{GW}$. The same transmission can be fit using the FD model and NLA values measured from this work. After scaling for wavelength using Eq. (1), $\beta=30 \mathrm{~cm} / \mathrm{GW}$ and $\sigma=8 \times 10^{-16} \mathrm{~cm}^{2}$ provide an excellent fit, highlighting the need for a simultaneous $\beta$ and $\sigma$ measurement technique.

Figure 4 (left) shows the $2.05 \mu \mathrm{m}$ Ge nanosecond NLA data (red circles) and picosecond NLA data (blue circles), along with the theoretical fitting lines obtained from the best fit pair of $\beta=71 \mathrm{~cm} / \mathrm{GW}$ and $\sigma=4.9 \times 10^{-16} \mathrm{~cm}^{2}$. The quantitative fit to both picosecond and nanosecond data is shown in Fig. 4 (right), which plots the inverse sum of squared error resulting from a spectrum of $\beta$ and $\sigma$. Regions of best fit are shown in red, with a yellow star signifying the best quantitative fit, which reveals the true values of $\beta$ and $\sigma$. The theoretical lines on the left chart result from these values, showing that an excellent fit was obtained.

Using the same approach, the dual pulse width NLA data that were collected for $2.05 \mu \mathrm{m}$ GaSb, $2.5 \mu \mathrm{m} \mathrm{Ge}$, and $2.5 \mu \mathrm{m}$ GaSb were fit using either Gaussian or gainswitched temporal profiles in the FD model, as appropriate. A two step method was used to calculate the uncertainty in reported $\beta$ and $\sigma$ values. First, a propagation of errors method was used to calculate the uncertainty in the peak pulse irradiance that results from inaccuracies in pulse energy, pulse width, and spot size. Using this method, the irradiance uncertainty $\Delta I$ was calculated for each of the four experiments, which ranged from $9 \%-15 \%$.

The second step was to determine how $\Delta I$ affects $\beta$ and $\sigma$. The uncertainty range was measured by scaling the peak irradiance of the NLA data by $\pm \Delta I$ and then refitting using the quantitative technique described earlier. The fit is sensitive to errors in the nanosecond and picosecond data, yielding four combinations of errors that are tested: $\pm \mathrm{ns} I$ and $\pm \mathrm{ps} I$. In these four scenarios, it was noted that there was $10 \%-15 \%$ variation in $\beta$ and $\sigma$ when the nanosecond and picosecond data are scaled in the same direction; however greater variation resulted when one data set was scaled with the opposite sign. The error range for the coefficients presented here is not symmetric, which was unsurprising due to the nonlinear processes involved. The measured NLA coefficients and uncertainty ranges are summarized in Table 4 . In the table, the bounds are

Table 4. NLA Coefficient Measurements at Two Mid-IR Wavelengths for Ge and GaSb

\begin{tabular}{lccccc}
\hline & \multicolumn{2}{c}{$\mathrm{Ge}$} & & \multicolumn{2}{c}{$\mathrm{GaSb}$} \\
\cline { 2 - 3 } \cline { 5 - 6 } Wavelength $(\mu \mathrm{m})$ & 2.05 & $2.4-2.51$ & & 2.05 & $2.4-2.51$ \\
\hline$\beta(\mathrm{cm} / \mathrm{GW})$ & 71 & 68 & 64 & 119 \\
(lower-upper bound) & $(45-96)$ & $(57-95)$ & & $(49-90)$ & $(86-148)$ \\
$\sigma\left(10^{-16} \mathrm{~cm}^{2}\right)$ & 4.9 & 9.0 & & 3.8 & 6.5 \\
(lower-upper bound) & $(3.0-9.4)$ & $(5.7-12.5)$ & & $(2.5-5.4)$ & $(5.0-9.8)$ \\
\hline
\end{tabular}



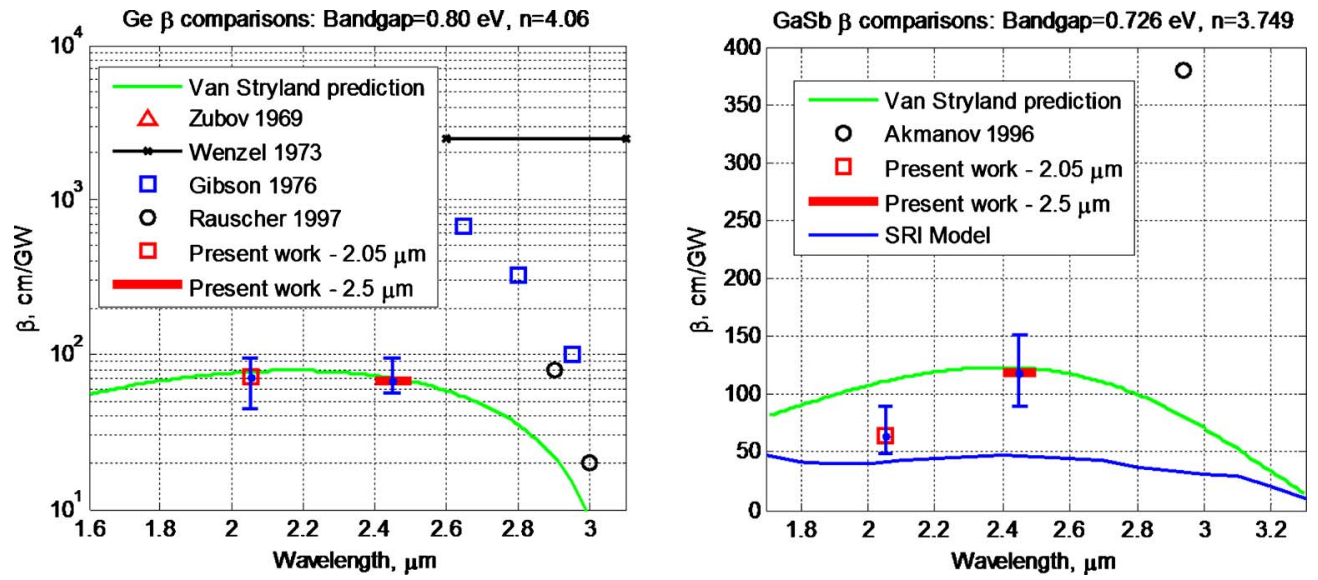

Fig. 5. (Color online) Wavelength dependence of $\beta$ in present work, literature, and theory for Ge (left) and GaSb (right).

defined as the minimum and maximum $\beta$ and $\sigma$ that result from refitting at $\pm \mathrm{ns} \Delta I$ and $\pm \mathrm{ps} \Delta I$.

The NLA coefficients reported in Table 4 are now compared to literature values and theoretical predictions. $\beta$ measurements are compared in Fig. 5, where the left graph compares the measured Ge $\beta$ with literature values, and the theory from Eq. (1) is overlaid as the green trace. The Ge values from this work are in excellent agreement with the prediction and are in reasonable agreement with the only literature value that also used FCA in their NLA fit [15], even though their $\sigma$ value was an estimate.

GaSb $\beta$ are compared in Fig. 5 (right), and the $2.5 \mu \mathrm{m}$ value is in excellent agreement with Eq. (1). The discrepancy between the measured GaSb $2.05 \mu \mathrm{m}$ value and the Van Stryland prediction of $\beta=111 \mathrm{~cm} / \mathrm{GW}$ may be explained with a GaSb full band-structure prediction created by SRI International that predicts a $2.05 \mu \mathrm{m} \beta$ of 41 $\mathrm{cm} / \mathrm{GW}$ [10]. This model is overlaid as the blue line in Fig. 5 (right) and is in better agreement with the value reported in this work. It is noted that neither model provides an excellent fit to both data points; however the uncertainty range of each model may intersect the uncertainty range of the reported values. The 1996 study of Akmanov et al. [16] at $2.94 \mu \mathrm{m}$ used an estimate of $\sigma=2 \times 10^{-17} \mathrm{~cm}^{2}$ which is 1 order of magnitude smaller than the values reported in this work $\left(3.8 \times 10^{-16} \mathrm{~cm}^{2}\right.$ and $6.5 \times 10^{-16} \mathrm{~cm}^{2}$ ). This results in a much higher fit to $\beta$, which is expected due to the inverse relationship between $\beta$ and $\sigma$ that is visible in Fig. 4 (right). It was not possible to fit the transmission data from the 1996 study of Akmanov et al. [16] using measured $\sigma$ values from this work as the pulse width, level of linear transmission, spot size, and spatial profile were not reported.

\section{A. Laser-Induced Damage Study}

The culmination of this study was the measurement and modeling of single-pulse LIDTs in Ge and GaSb at $2.5 \mu \mathrm{m}$ that result from NLA, $\tau_{R}(N)$, and $\alpha(T)$. While the coatings were applied for the nonlinear measurements, the damage threshold of the coated Ge sample was measured as an additional data point.

Picosecond damage tests: The source for experiment $\mathrm{V}$ was the tunable Ekspla DFG that was also used in the NLA testing. For this test, the pulse width was previously measured as $10.1 \pm 0.83 \mathrm{ps}$ using an autocorrelator at $2.5 \mu \mathrm{m}$. For the GaSb and Ge picosecond LIDT experiments, 44 and $98 \mu \mathrm{m} r_{o}$ 's were measured, respectively, and both possessed near-perfect Gaussian spatial profiles. The lens had a tight focus, and the spot radius was highly dependent on $z$ position, and the sample was accurately placed at the focus using an infrared focal plane array (FPA). First, the pinhole was located at the focus using a series of raster scans, and the pinhole was imaged on the FPA. Then, the pinhole would be swapped with the sample, and the sample $z$ position would be altered until the sample was in focus. The sample would become in or out of focus at a $\Delta z$ of $100 \mu \mathrm{m}(0.1 \mathrm{~mm})$, allowing very accurate placement. The process was aided using a soldering iron as a "thermal flashlight" to bring out details on the pinhole or sample surface on which to focus.

The damage test plan was guided by ISO 11254-1, which governs single shot laser-induced damage testing [39]. The single shot test plan included ten sites per fluence level, with site spacing of two to three times beam diameter. The incident beam was horizontally polarized, which corresponds to a left-right orientation in Figs. 6 and 8 . Ten shot coated Ge morphologies are presented in Fig. 6 with increasing levels of fluence $(F)$.

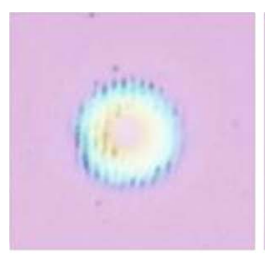

a) $\mathrm{F}=14 \mathrm{~mJ} / \mathrm{cm}^{2}$

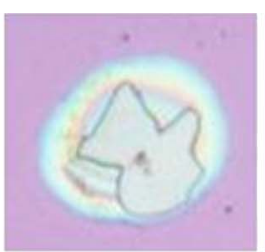

b) $\mathrm{F}=21 \mathrm{~mJ} / \mathrm{cm}^{2}$

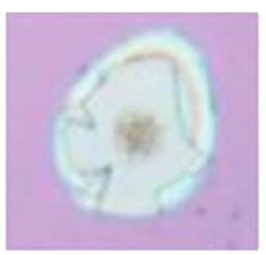

c) $F=31 \mathrm{~mJ} / \mathrm{cm}^{2}$

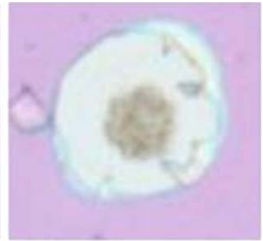

d) $\mathrm{F}=37 \mathrm{~mJ} / \mathrm{cm}^{2}$

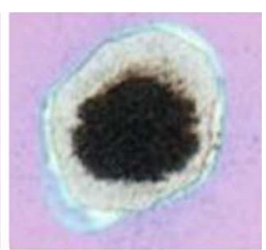

e) $\mathrm{F}=82 \mathrm{~mJ} / \mathrm{cm}^{2}$

Fig. 6. (Color online) Evolution of ten shot per site damage in coated germanium at $2.5 \mu \mathrm{m}$ using picosecond pulses. 


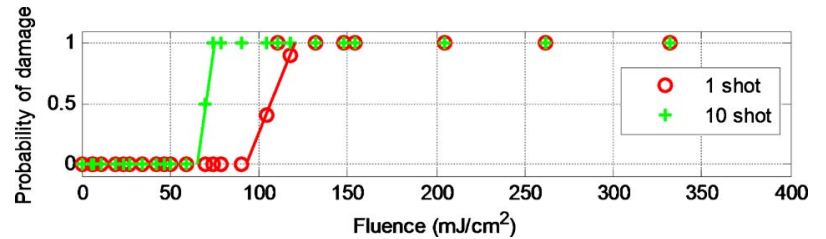

Fig. 7. (Color online) Picosecond LIDT measurement for uncoated GaSb, using ISO-11254-1 method.

Very close to threshold in Fig. 6(a)), the coating was ablated slightly, but there was no damage to the Ge surface, demonstrating that the coating has a lower damage threshold. The coating reduced the Fresnel surface reflection to $5 \%$ from $37 \%$, allowing a greater irradiance to be absorbed in the skin depth of the sample. This could possibly lower the surface LIDT; however it was clear that the coating has a lower damage threshold as the coating could damage without underlying surface damage. GaSb damage morphology was similar to Ge, although higher fluence levels were required to achieve damage.

Using the ISO 11254-1 method, the single shot threshold for uncoated GaSb is measured in Fig. 7 to be $93 \mathrm{~mJ} / \mathrm{cm}^{2}$, and the ten shot threshold is $65 \mathrm{~mJ} / \mathrm{cm}^{2}$. The picosecond single shot LIDTs for coated and uncoated $\mathrm{Ge}$ are measured in the same manner and are reported in Table 5, along with 10- and 30-shot LIDTs.

Nanosecond damage testing: In experiment VI, singlepulse surface LIDT tests were conducted on GaSb, Ge, and coated Ge at $2.5 \mu \mathrm{m}$ using nanosecond pulses from the gain-switched $\mathrm{Cr}^{2+}: \mathrm{ZnSe}$ laser. In the nanosecond tests, the Ge damage morphology did not exhibit dramatic scarring or boiling, only surface melting. For coated Ge, at fluences very close to the threshold of $1.25 \mathrm{~J} / \mathrm{cm}^{2}$, the coating was ablated slightly without underlying surface damage, confirming the observation from the picosecond coated damage test. The lower melting point and specific heat of GaSb caused greater surface modifications than found in Ge as shown in Fig. 8 for single shot damage sites. At a fluence near threshold, a series of thermal fractures are visible in Fig. 8(a) which then proceed to smoothly melt at higher fluences.

All damage threshold measurements are presented in Table 5 for uncoated GaSb, uncoated Ge, and coated Ge along with parameters characterizing the damage tests. The uncertainty in the reported LIDT fluence resulting from uncertainties in $r_{o}$ and pulse energy measurement was calculated using the same propagation of errors method as the NLA coefficients.

\section{B. Modeling of Damage Thresholds}

Pulse width $\left(\tau_{p}\right)$ scaling of the LIDT typically possesses a $\tau_{p}{ }^{x}$ dependence where $x$ ranges from 0.4-0.5 [40], which was confirmed with reported LIDT values and the modeling in Fig. 9. When using a $\tau_{p}^{0.5}$ value of pulse width scaling, the Ge $\tau_{p}=10 \mathrm{ps}$ LIDT measurement was in excellent agreement with the $\tau_{p}=68 \mathrm{~ns}$ LIDT $\left(1.94 \mathrm{~J} / \mathrm{cm}^{2}\right.$ versus $1.85 \mathrm{~J} / \mathrm{cm}^{2}$ predicted). Using this method, GaSb had a $\tau_{p}{ }^{0.34}$ dependence on the pulse width. An iteration routine was used with the FD model to find the pulse fluence that will cause surface melting, and Fig. 9 presents measured single pulse LIDTs (picosecond and nanosecond) for these two materials overlaid with predicted thresholds from the FD model. These predictions use no fitting parametersonly measured NLA coefficients, measured beam parameters, carrier density dependent $\tau_{R}(N)$ from the literature, and measured $\alpha(T)$. The calculated error bars are smaller than the symbol shapes due to the log-log presentation.

The range of pulse widths in Fig. 9 was bounded by free-carrier diffusion and dielectric breakdown, which are effects not included in the FD model. A free-carrier diffusion length of $100 \mu \mathrm{m}$ was selected as the constraint on the longest pulse width modeled, which corresponds to a pulse width of 500 (350) ns for Ge (GaSb). As the pulse width is decreased toward the femtosecond regime, eventually a transition to dielectric breakdown will occur. The shortest pulse width modeled corresponds to the predicted Keldysh theory transition [41,42], which was on the order of $\tau_{p}=300 \mathrm{fs}$ for these materials. $\% T$ designates linear transmission through the uncoated samples as measured on a spectrophotometer (Varian, Cary 5000). As shown by the dotted lines in Fig. 9, the inclusion of $\alpha(T)$ lowers the predicted LIDT by a factor of 2 for $\tau_{p}$ near $1 \mu \mathrm{s}$, and only affects pulses longer than $100 \mathrm{ps}$.

The LIDT values are in good agreement with theory with one exception-at a picosecond pulse width, Ge damaged at a fluence level five times less than predicted. Possible causes for this discrepancy include field enhancement and thermalization, but not dielectric breakdown, which is predicted only if the irradiance were increased by 1 order of magnitude. The incident electric field at surface defects is known to be locally enhanced at a rate proportional to $n^{4}$ [31], giving Ge a 35\% greater enhancement over GaSb. This calculated enhancement is only based on the difference in $n$, and the $20 \%$ greater surface roughness of Ge will further magnify the field. While the hotcarrier thermalization of Ge was not modeled as it was beyond the scope of this study, its non-equilibrium phonon

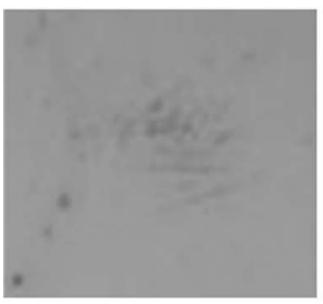

a) $\mathrm{F}=1.9 \mathrm{~J} / \mathrm{cm}^{2}$

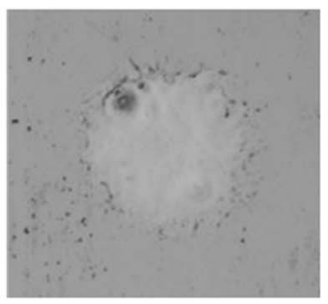

b) $\mathrm{F}=2.7 \mathrm{~J} / \mathrm{cm}^{2}$

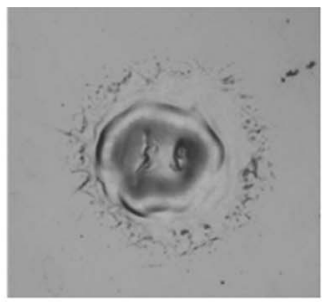

c) $\mathrm{F}=2.9 \mathrm{~J} / \mathrm{cm}^{2}$

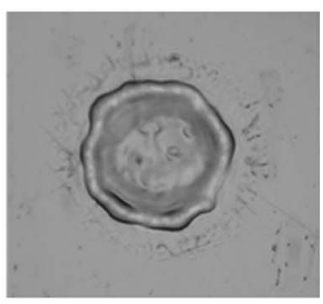

d) $\mathrm{F}=3.2 \mathrm{~J} / \mathrm{cm}^{2}$

Fig. 8. Evolution of single shot damage in uncoated GaSb at $2.5 \mu \mathrm{m}$ using nanosecond pulses. 
Table 5. Results from 2.5 $\mu \mathrm{m}$ Damage Study, Including Key Parameters

\begin{tabular}{|c|c|c|c|c|c|c|}
\hline Sample & Pulse Width & Shots per Site & $\begin{array}{c}\text { LIDT } \\
\left(\mathrm{mJ} / \mathrm{cm}^{2}\right)\end{array}$ & $\begin{array}{l}\text { Spot Radius } \\
(\mu \mathrm{m})\end{array}$ & $\begin{array}{c}\text { Peak } I \text { at LIDT } \\
\left(\mathrm{GW} / \mathrm{cm}^{2}\right)\end{array}$ & $\begin{array}{c}E \text { at LIDT } \\
(\mu \mathrm{J})\end{array}$ \\
\hline GaSb uncoated & $10.1 \mathrm{ps}$ & 1 & $93 \pm 15$ & 44 & 4.97 & 5.5 \\
\hline GaSb uncoated & $10.1 \mathrm{ps}$ & 10 & $65 \pm 12$ & 44 & 3.36 & 3.7 \\
\hline Ge uncoated & $10.1 \mathrm{ps}$ & 1 & $24 \pm 3.2$ & 98 & 1.24 & 6.8 \\
\hline Ge uncoated & $10.1 \mathrm{ps}$ & 10 & $18 \pm 2.7$ & 98 & 0.932 & 5.1 \\
\hline Ge uncoated & $10.1 \mathrm{ps}$ & 30 & $15 \pm 2.4$ & 98 & 0.776 & 4.2 \\
\hline $\mathrm{Al}_{2} \mathrm{O}_{3}$ coating on $\mathrm{Ge}$ & $10.1 \mathrm{ps}$ & 1 & $11 \pm 2.1$ & 98 & 0.569 & 3.1 \\
\hline $\mathrm{Al}_{2} \mathrm{O}_{3}$ coating on $\mathrm{Ge}$ & $10.1 \mathrm{ps}$ & 10 and 30 & $10 \pm 2.0$ & 98 & 0.517 & 2.8 \\
\hline GaSb uncoated & $68 \mathrm{~ns}^{a}$ & 1 & $1750 \pm 170$ & 136 & 0.015 & 1020 \\
\hline Ge uncoated & $68 \mathrm{~ns}^{a}$ & 1 & $1940 \pm 180$ & 136 & 0.017 & 1130 \\
\hline $\mathrm{Al}_{2} \mathrm{O}_{3}$ coating on $\mathrm{Ge}$ & $68 \mathrm{~ns}^{a}$ & 1 & $1250 \pm 140$ & 136 & 0.011 & 730 \\
\hline
\end{tabular}

${ }^{a}$ Gain-switched pulse.

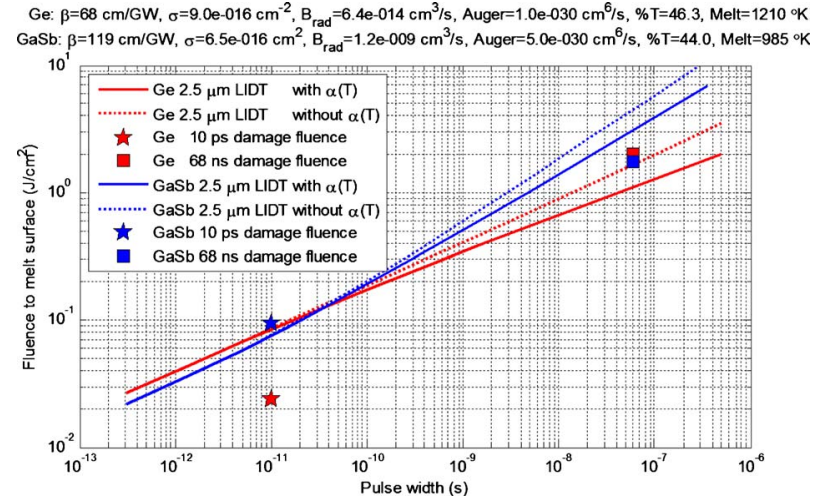

Fig. 9. (Color online) Predicted LIDT fluence for uncoated Ge and GaSb using measured NLA coefficients. Markers indicate experimentally determined LIDT fluences, solid lines are modeling that includes $\alpha(T)$, and dotted lines model LIDT without $\alpha(T)$.

decay time of 4 ps could certainly contribute toward a lower LIDT for a 10 ps pulse width [43].

\section{CONCLUSIONS}

NLA coefficients ( $\beta$ and $\sigma$ ) in Ge and GaSb at both 2.05 and $2.5 \mu \mathrm{m}$ were reported, which were measured using a simultaneous fit method and a finite difference (FD) model developed in this work. Pulsed damage threshold modeling was performed using measured NLA coefficients and measured $\alpha(T)$, and modeling was verified with ten measurements of the LIDT across two pulse widths. In a regime where NLA is dominant, the inclusion of $\alpha(T)$ lowers the predicted LIDT by a factor of 2 for $\tau_{p}$ near $1 \mu \mathrm{s}$, and only affects pulses longer than $100 \mathrm{ps}$ in these materials. Finally, a single-layer $\mathrm{Al}_{2} \mathrm{O}_{3}$ anti-reflective coating on Ge was found to possess a lower LIDT than the uncoated surface for both picosecond and nanosecond pulses.

\section{ACKNOWLEDGMENTS}

The authors would like to thank R. Shori (University of California, Los Angeles) and A. Sarangan (University of Dayton) for material support to these experiments. The authors would also like to thank their colleagues and collaborators P. Berry, R. Peterson, J. McCurdy, D. Upchurch, A. Carpenter, and J. Barnes (Air Force Research
Laboratory-AFRL) as well as funding support from the Sensors Directorate of AFRL, the Materials and Manufacturing Directorate of AFRL and the U.S. Air Force Office of Scientific Research (AFOSR).

\section{REFERENCES}

1. T. H. Maiman, "Stimulated optical radiation in ruby," Nature 187, 493-494 (1960).

2. S. Mirov, V. Fedorov, I. Moskalev, D. Martyshkin, and C. Kim, "Progress in $\mathrm{Cr}^{2+}$ and $\mathrm{Fe}^{2+}$ doped mid-IR laser materials," Laser Photonics Rev. 4, 21-41 (2010).

3. M. Göppert-Mayer, "über Elementarakte mit zwei Quantensprüngen," Ph.D. dissertation (University of Göttingen, 1931).

4. W. Kaiser and C. G. B. Garrett, "Two-photon excitation in $\mathrm{CaF}_{2}: \mathrm{Eu}^{2+}$," Phys. Rev. Lett. 7, 229-231 (1961).

5. M. Sheik-Bahae and M. P. Hasselbeck, "Third-order optical nonlinearities," in OSA Handbook of Optics (McGraw-Hill, 2001), Vol. 4, Chap. 17.

6. D. N. Christodoulides, I. C. Khoo, G. J. Salamo, G. I. Stegeman, and E. W. Van Stryland, "Nonlinear refraction and absorption: mechanisms and magnitudes," Adv. Opt. Photon. 2, 60-200 (2010)

7. B. S. Wherrett, "Scaling rules for multiphoton interband absorption in semiconductors," J. Opt. Soc. Am. B 1, 67-72 (1984).

8. E. W. Van Stryland, M. A. Woodall, H. Vanherzeele, and M. J. Soileau, "Energy band-gap dependence of two-photon absorption," Opt. Lett. 10, 490-492 (1985).

9. E. Tuncel, J. L. Staehli, C. Coluzza, G. Margaritondo, J. T. McKinley, R. G. Albridge, A. V. Barnes, A. Ueda, X. Yang, and N. H. Tolk, "Free-electron laser studies of direct and indirect two-photon absorption in germanium," Phys. Rev. Lett. 70, 4146-4149 (1993).

10. S. Krishnamurthy, SRI International, 333 Ravenswood Avenue, Menlo Park, Calif. (personal communication, 2010).

11. S. Krishnamurthy, Z. G. Yu, S. Guha, and L. Gonzalez, "High irradiance light propagation in InAs," Appl. Phys. Lett. 89, 161108 (2006).

12. B. V. Zubov, L. A. Kulevskii, V. P. Makarov, T. M. Murina, and A. M. Prokhorov, "Two-photon absorption in germanium," J. Exp. Theor. Phys. 9, 130-132 (1969).

13. R. G. Wenzel, G. P. Arnold, and N. R. Greiner, "Nonlinear loss in Ge in the 2.5-4- $\mu \mathrm{m}$ range," Appl. Opt. 12, 22452247 (1973)

14. A. F. Gibson, C. B. Hatch, P. N. D. Maggs, D. R. Tilley, and A. C. Walker, "Two-photon absorption in indium antimonide and germanium," J. Phys. C 9, 3259-3275 (1976).

15. C. Rauscher and R. Laenen, "Analysis of picoseconds midinfrared pulses by two-photon absorption in germanium," J. Appl. Phys. 81, 2818-2821 (1997).

16. A. G. Akmanov, B. V. Zhdanov, and B. G. Shakirov, "Twophoton absorption of IR radiation and its optical saturation 
in n-type gallium antimonide," Quantum Electron. 26, 882883 (1996).

17. M. D. Turner, W. B. Roh, and K. L. Schepler, "Nonlinear optical properties of GaSb and GaInAsSb and their application for phase conjugation in degenerate four-wave mixing," J. Opt. Soc. Am. B 17, 790-804 (2000).

18. D. A. Reis, K. J. Gaffney, G. H. Gilmer, and B. Torralva, "Ultrafast dynamics of laser-excited solids," MRS Bull. 31, 601-606 (2006)

19. G. E. Jellison, Jr., D. H. Lowndes, D. N. Mashburn, and R. F. Wood, "Time-resolved reflectivity measurements on silicon and germanium using a pulsed excimer $\mathrm{KrF}$ laser heating beam," Phys. Rev. B 34, 2407-2415 (1986).

20. D. Seo, L. C. Feldman, N. H. Tolk, and P. I. Cohen, "Interaction of high-power infrared radiation with germanium," Proc. SPIE 7132, 713216 (2008).

21. J. B. McKay, "Power scaling feasibility of chromium-doped II-VI laser sources and the demonstration of a chromiumdoped zinc selenide disk Laser," Ph.D. dissertation (Air Force Institute of Technology, 2002).

22. T. J. Carrig, G. J. Wagner, W. J. Alford, and A. Zakel, "Chromium-doped chalcogenide lasers," Proc. SPIE 5460, 74-82 (2004)

23. T. R. Harris, "Optical properties of Si, Ge, GaAs, GaSb, InAs, and InP at elevated temperatures," Master's thesis (Air Force Institute of Technology, 2010).

24. R. N. Hall, "Electron-hole recombination in germanium," Phys. Rev. 87, 387 (1952).

25. E. Marin, I. Riech, P. Diaz, J. J. Alvarado-Gil, R. Baquero, J. G. Mendoza-Alvarez, H. Vargas, A. Cruz-Orea, and M. Vargas, "Photoacoustic determination of non-radiative carrier lifetimes," J. Appl. Phys. 83, 2604-2609 (1998).

26. M. Levinstein, S. Rumyantsev, and M. Shur, Handbook Series on Semiconductor Parameters (World Scientific, 1996 , 1999); available online at http://www.ioffe.ru/SVA/NSM/ Semicond.

27. M. D. Dramićanin, P. M. Nikoli, Z. D. Ristovski, D. G. Vasiljevi, and D. M. Todorovi, "Photoacoustic investigation of transport in semiconductors: Theoretical and experimental study of a Ge single crystal," Phys. Rev. B 51, 14226-14232 (1995).

28. P. S. Dutta, H. L. Bhat, and V. Kumar, "The physics and technology of gallium antimonide: An emerging optoelectronic material," J. Appl. Phys. 81, 5821-5870 (1997).

29. R. A. Dragoset, C. W. Clark, W. C. Martin, P. J. Mohr, and B. N. Taylor, Periodic Table: Atomic Properties of the Elements (National Institute of Standards and Technology, 1999).

30. J. C. Phillips and J. A. Van Vechten, "Dielectric classification of crystal structures, ionization potentials, and band structures," Phys. Rev. Lett. 22, 705-708 (1969).
31. R. M. Wood, Laser-induced Damage of Optical Materials (Taylor \& Francis, 2003), pp. 31-32.

32. S. J. Sheldon, L. V. Knight, and J. M. Thorne, "Laserinduced thermal lens effect: a new theoretical model," Appl. Opt. 21, 1663-1669 (1982).

33. L. P. Gonzalez, J. M. Murray, V. M. Cowan, and S. Guha, "Measurement of the nonlinear optical properties of semiconductors using the irradiance scan technique," Proc. SPIE 6875, 68750R (2008).

34. V. Nathan, A. H. Guenther, and S. S. Mitra, "Review of multiphoton absorption in crystalline solids," J. Opt. Soc. Am. B 2, 294-316 (1985)

35. O. A. Louchev, Y. Urata, and S. Wada, "Numerical simulation and optimization of giant pulse generation in 2 microns Tm,Ho lasers," in Conference on Lasers and Electro-Optics / Quantum Electronics and Laser Science Conference and Photonic Applications Systems Technologies, OSA Technical Digest Series (CD) (Optical Society of America, 2007), paper CThDD2.

36. O. A. Louchev, Y. Urata, N. Saito, and S. Wada, "Computational model for operation of $2 \mu \mathrm{m}$ co-doped Tm,Ho solidstate lasers," Opt. Express 15, 11903-11912 (2007).

37. L. P. Gonzalez, J. M. Murray, S. Krishnamurthy, and S. Guha, "Wavelength dependence of two photon and free carrier absorptions in InP," Opt. Express 17, 8741-8748 (2009).

38. T. J. Sloanes, "Measurement and application of optical nonlinearities in indium phosphide, cadmium mercury telleride and photonic crystal fibres," Ph.D. dissertation (University of St. Andrews, 2009).

39. International Organization for Standardization (IOS), Lasers and Laser-Related Equipment-Determination of Laser-Induced Damage Threshold of Optical SurfacesPart 1: 1-on-1 Test (IOS, 2000).

40. B. C. Stuart, M. D. Feit, A. M. Rubenchik, B. W. Shore, and M. D. Perry, "Laser-induced damage in dielectrics with nanosecond to subpicosecond pulses," Phys. Rev. Lett. 74, 2248-2251 (1995).

41. L. V. Keldysh, "Ionization in the field of a strong electromagnetic wave," Sov. Phys. JETP 20, 1307-1314 (1965).

42. A. P. Joglekar, H. Liu, G. J. Spooner, E. Meyhöfer, G. Mourou, and A. J. Hunt, "A study of the deterministic character of optical damage by femtosecond laser pulses and applications to nanomachining," Appl. Phys. B 77, 25-30 (2003).

43. A. Othonos, H. M. Van Driel, J. F. Young, and P. J. Kelly, "Correlation of hot-phonon and hot-carrier kinetics in Ge on a picosecond time scale," Phys. Rev. B 43, 6682-6690 (1991). 